\title{
Foreword: special issue on coalgebraic logic
}

\author{
ERNST-ERICH DOBERKAT ${ }^{\dagger}$ and ALEXANDER KURZ \\ ${ }^{\dagger}$ Chair for Software Technology, Technische Universität Dortmund, Germany \\ Email: ernst-erich.doberkat@udo.edu

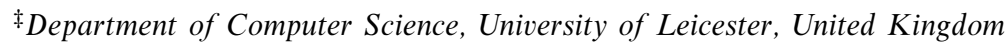 \\ Email: kurz@mcs.le.ac.uk
}

Received 30 September 2010

The Coalgebraic Logic seminar took place between the 6th and 9th of December 2009 at the Leibniz Forschungszentrum Schloss Dagstuhl. The event was very well received, with more than 35 scientists from Europe, the United States, Canada and China attending, and with more than thirty presentations during the three days. Given the unforeseen enthusiasm of the meeting's reception, we thought that it would be a good idea for us to put together a special journal issue dedicated to this topic. We were also encouraged by the positive response we received from Guiseppe Longo, who indicated his interest in a special issue for Mathematical Structures in Computer Science, and here we are.

\section{Coalgebraic logics}

Modal logic is a field with roots in philosophical logic and mathematics. In its applications to computer science, it has become central for reasoning about the behavioural and temporal properties of computing and communicating systems, and for modelling properties of agents such as knowledge, obligations and permissions. It is remarkable that many modal logics are, despite their considerable expressive power, decidable and, therefore, amenable to automated reasoning and verification. Moreover, Kripke's relational semantics of modal logic has turned out to be amazingly flexible, both in terms of providing techniques for proving properties of modal logics and for allowing various applications of modal logic to artificial intelligence, software agents and many other fields.

Coalgebras generalise Kripke semantics. Following on from Aczel's seminal work on non-well-founded set theory, coalgebra has been developed into a general theory of systems. The basic idea is that coalgebras are given with respect to a parameter $\mathbf{F}$, which is a functor on a category $\mathscr{C}$. Varying the functor $\mathbf{F}$ allows us to model different features such as input, output, choice, non-determinism, predicate transformers and probability. Varying the category $\mathscr{C}$ allows us to equip the set of states of the generalised transition systems with additional structure. This can be a topology for modelling a notion of observable predicate, or an algebra for modelling, for example, programming constructs from process algebra.

Different choices of $\mathbf{F}$ yield, for example, the Kripke frames and models of modal logic, the labelled transition systems of process algebra, the deterministic automata of formal

†Partially supported by EPSRC EP/G041296/1. 
language theory, or the Markov chains used in statistics. Rutten showed in his well-known survey that, by analogy with Universal Algebra, a theory of systems, called Universal Coalgebra, can be built uniformly in the parameter $\mathbf{F}$, simultaneously covering the above and other examples. Crucial notions such as behavioural equivalence, observational equivalence, bisimilarity, final semantics and coinduction find their natural place here.

Coalgebraic Logic combines Coalgebra and Modal Logic to study logics of systems uniformly in the parameter F. Given the abundance of different transition systems and their ad hoc logics, such a uniform theory is clearly desirable. Here, 'uniformity' means that results on, for example, completeness, expressivity, the finite model property and the complexity of satisfiability can be established at the same time for all functors, usually satisfying some, usually mild, conditions.

There is also a concern for modularity. Typically, a parameter $\mathbf{F}$ is composed of basic features such as input, output, non-determinism and probability. Modularity then means that the syntax (proof systems, algorithms) of the logic of $\mathbf{F}$ are obtained compositionally from the syntax (proof systems, algorithms) of the logics of the basic features.

\section{The contributions}

We conclude this introduction with a brief overview of the papers included in this special issue.

In their paper Initial algebras and terminal coalgebras in many-sorted sets, J. Adámek and V. Trnková discuss the iterative construction of initial algebras and final coalgebras for endofunctors on many-sorted sets. In particular, they generalise the result known for the one-sorted case that this construction converges whenever the endofunctor has an initial/final algebra. They also show, maybe somewhat surprisingly, that this result cannot be further extended from many-sorted sets to presheaf categories.

The paper Elgot theories: a new perspective on the equational properties of iteration by J. Adámek, S. Milius and J. Velebil deals with an extension to Bloom and Ésik's concept of iteration theory, which summarises all the equational properties that iteration has in normal applications. It shows that in the general coalgebraic approach to iteration, the corresponding concept is that of a functorial iteration theory. Functoriality here refers to an axiom that was not included in Bloom and Ésik's concept of an iteration theory, since its form is implicational and not equational. One of the surprising contributions of this paper is the revelation that functoriality is equational if 'equational' is taken in the enriched sense of Kelly and Power.

Going beyond the context of set-based functors, the paper Coalgebraic logic over general measurable spaces - a survey by E.-E. Doberkat and Ch. Schubert discusses the generalisation of stochastic Kripke models for general modal logics through predicate liftings for functors over general measurable spaces. The paper derives results on expressivity and shows that selection arguments enable the discussion of bisimilarity, provided the underlying spaces are Polish.

The paper Traces for coalgebraic components by I. Hasuo and B. Jacobs continues earlier work by P. Katis, N. Sabadini, R. Walters and L. Barbosa on calculi for state-based components. In particular, it adds a feedback operator in the form of a monoidal trace 
and develops a three-dimensional string calculus to represent and manipulate composite component diagrams.

H. H. Hansen and B. Klin's paper Pointwise extensions of GSOS-defined operations continues a line of research initiated by Turi and Plotkin with the observation that algebraic operations on elements of a final coalgebra can be defined by distributive laws of a syntax functor over a behaviour functor; moreover, distributive laws correspond to abstract specification formats such as the GSOS format known from process algebra. The paper shows that, given an abstract GSOS specification for a behaviour functor $\mathbf{F}$, one can systematically construct a GSOS specification for the pointwise extension on the final $\mathbf{F}^{A}$-coalgebra. The paper provides many examples illustrating the constructions and showing their relevance.

The paper On monotone modalities and adjointness by M. Bílková, J. Velebil, and Y. Venema contributes to the study of a particular coalgebraic logic introduced in a seminal 1997 paper by L. Moss. Formulas of this logic $\mathscr{L}$ are built from a 'non-standard' modality $\nabla$, which constructs formulas $\nabla \alpha$ for $\alpha \in \mathbf{F} \mathscr{L}$, where $\mathbf{F}$ is the set-functor determining the semantics in terms of coalgebras $X \rightarrow \mathbf{F} X$. The paper shows that $\nabla: \mathbf{F} \mathscr{L} \rightarrow \mathscr{L}$ has a right adjoint in the weaker sense that there is $G: \mathscr{L} \rightarrow \mathscr{P}_{\omega}(\mathbf{F} \mathscr{L})$ such that $\nabla \alpha \leqslant b$ if and only if $\alpha \leqslant \gamma$ for some $\gamma \in G(b)$. The proof makes innovative use of the dual operator $\Delta$ of $\nabla$. The paper spells out the consequences of its results for 'standard' unary modalities.

In their paper Modular algorithms for heterogeneous modal logics via multi-sorted coalgebra, L. Schröder and D. Pattinson address the combining of features, such as nondeterminism and probabilities, for modelling state-based systems. They show that the combination can be reflected algorithmically, and develop modular decision procedures for heterogeneous modal logics. The modularity is achieved by formalising the underlying state-based systems as multi-sorted coalgebras and associating both a logical and an algorithmic description with a number of basic building blocks. The main result is that logics arising as combinations of these building blocks can be decided in polynomial space, provided this is also the case for the components.

In their paper Equational presentations of functors and monads, J. Velebil and A. Kurz generalise the result (from earlier work with J. Rosický) that all 'universal-algebraic' endofunctors $L$ on a variety $\mathscr{K}$ have a presentation by operations and equations. To this end, the notion of a functor $L$ being based is introduced, and it is shown that based functors can be presented in the more general situation where $\mathscr{K}$ is not a variety but simply of descent type. Moreover, building on work by Kelly and Power, this result transfers from set-based universal algebra to the enriched setting, and from functors to monads.

\section{Acknowledgements}

We are grateful to the Leibniz Forschungszentrum Schloss Dagstuhl and its very helpful staff for organising and hosting the Coalgebraic Logic seminar in December 2009; in particular, we wish to thank Ingo Battenfeld (Dortmund) for the assistance he gave us as organisers. Dagstuhl, as it is affectionately known to computer scientists, is a truly wonderful place for meeting people and exchanging research ideas. 
We would like to thank the authors and referees for their quick and efficient work, and although we did not accept all the submitted papers, we would like to extend our thanks to all the authors and referees who made this issue possible. Professor Longo, the Editor-in-Chief of Mathematical Structures in Computer Science, has been very supportive of this special issue, and we thank him for his continuous encouragement and support, not least for taking editorial responsibility for the two contributions co-authored by ourselves.

Special issue editors:

E.-E. Doberkat and A. Kurz

Dortmund and Leicester, September 2010 